\title{
Systematics of stone geckos in the genus Diplodactylus (Reptilia: Diplodactylidae) from northwestern Australia, with a description of a new species from the Northwest Cape, Western Australia
}

\author{
Paul Doughty', Paul Oliver ${ }^{2,3}$ and Mark Adams $s^{2,4,5}$ \\ 'Department of Terrestrial Zoology, Western Australian Museum, 49 Kew Street, \\ Welshpool Western Australia 6106, Australia. Email: Paul.Doughtymuseum.wa.gov.au \\ ¿South Australian Museum, North Terrace, Adelaide, South Australia 5000, Australia. \\ Email: Paul.Oliver adelaide.edu.au \\ ${ }^{4}$ Australian Centre for Evolutionary Biology and Biodiversity, University of Adelaide, \\ South Australia, 5005, Australia. \\ Email: Adams.Markesaugov.sa.gov.au
}

\begin{abstract}
Stone geckos (Diplodactylus spp.) are a small group of moderately robust geckos that occur on hard soils in southern and arid regions of Australia. We present a morphological and molecular assessment of the systematics of D. g. granariensis, D. g. rex and D. mitchelli, including an isolated population of $D$. 'mitchelli' from the Northwest Cape. Genetic data from allozyme analysis and 757 bp of the mitochondrial ND2 gene provided evidence for very recent divergence between the subspecies of $D$. granariensis, despite the morphological distinctiveness of $D$. g. rex from other stone geckos owing to large size, massive head, different pattern and scalation. In contrast, morphological and molecular data indicated that the isolated population of $D$. 'mitchelli' from the Northwest Cape was distinct at the species level and also more closely allied to $D$. granariensis. The new species differs from $D$. mitchelli in having smaller dorsal scales, fewer vertebrae and cloacal spurs, from D. g. granariensis in larger size, shorter tail and uncreased rostral scale, from $D$. g. rex by smaller body and head size and from both subspecies by fewer undivided subdigital lamellae, reddish colouration and distinctive dorsal stripe with transverse bands. The Northwest Cape has several other endemic species of reptiles indicating that this region is biogeographically significant and hence of special conservation value.
\end{abstract}

Keywords: Australia, Diplodactylus mitchelli, Diplodactylus g. granariensis, D. g. rex, Pilbara, wheatbelt

\section{INTRODUCTION}

The Australian continent has a diverse gecko fauna stemming from both ancient Gondwanan lineages (Carphodactylidae, Diplodactylidae and Pygopodidae) and more recent arrivals (Gekkonidae) (Han et al. 2004). Until recently the most speciose genus of Australian geckos was Diplodactylus. The only comprehensive taxonomic treatment of this group was by Kluge (1967), with significant taxonomic changes occurring since then. The arboreal spiny-tailed species were transferred to the genus Strophurus (Russell and Rosenberg 1984; Melville et al. 2004) and recently Oliver et al. (2007a) resurrected the genus Lucasium for the clade of more gracile, narrow-toed, long-tailed and highly terrestrial species based on molecular and morphological analyses. The genus Diplodactylus is now restricted to a group of species with a distinctive larger jugal, generally shorter tails and larger terminal scansors.
Within the redefined Diplodactylus there are thirteen recognized species (Cogger 2000; Wilson and Swan 2003; Bush et al. 2007). Recent genetic studies using mtDNA, however, have uncovered considerable evidence for high levels of unrecognized cryptic diversity (Oliver et al. 2007 a,b). Stone geckos of the genus Diplodactylus refer to a moderately robust terrestrial lineage of geckos with primarily a southern distribution. Currently recognized members in Western Australia include D. g. granariensis, D. g. rex, D. mitchelli, D. ornatus and D. polvophthalmus. Here we focus on the systematics of Western Australian stone geckos from northwestern and central Western Australia. These are D. mitchelli (Kluge 1963) from the Pilbara and presumably an isolated population from the Northwest Cape, D. g. granariensis (Storr 1979) from the wheatbelt and $D$. g. rex (Storr 1988) which occurs between the previous two taxa (Figure 1). Diplodactylus 


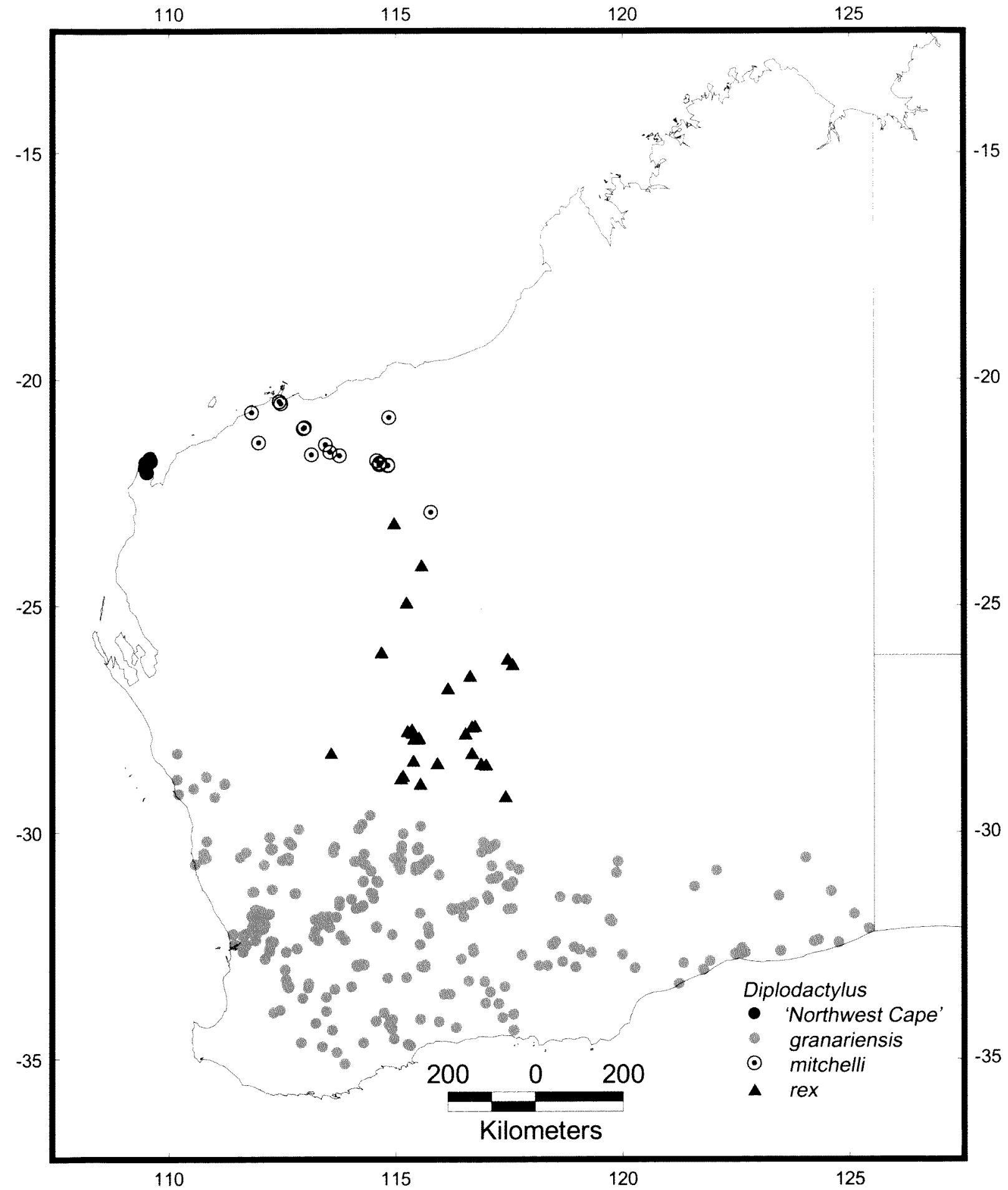

Figure 1 Distribution of Diplodactylus mitchelli, D. g. granariensis, D. g. rex and the isolated population of D. 'mitchelli' in Western Australia.

mitchelli is the most morphologically divergent of these taxa and can be readily diagnosed by its large size, long limbs, vertebral stripe with transverse bars on reddish background colour and enlarged dorsal scales (Storr et al. 1990; Cogger 2000). Diplodactylus granariensis from southwestern Australia was separated from the widespread $D$. vittatus by Storr (1979). Diplodactylus g. rex was described by Storr (1988) and diagnosed on the basis of its substantially larger size, uncreased rostral and distinctive pattern. At the time of its description, D. g. rex was believed to be allopatric from the nominate species. However, further collecting has shown the two taxa to be contiguous with morphology not always showing a clear demarcation between subspecies.

The isolated Diplodactylus population from the Northwest Cape was assigned to D. mitchelli by 
Kluge $(1963,1967)$, who noted that it differed in possessing smaller dorsal scales and restricted dorsal pattern. The genetic study of Oliver et al. (2007a) found that this population was not aligned to $D$. mitchelli, but instead was more closely related to D. granariensis. Limited sampling also indicated that there was little genetic support for the recognition of $D . g$. rex as distinct from $D . g$. granariensis. Here we use evidence from two different molecular techniques (mtDNA and allozymes) and morphology to elucidate the relationships and taxonomic status of the currently recognized taxa of northwestern stone geckos, with emphasis on the Northwest Cape population of $D$. mitchelli.

\section{METHODS}

\section{Materials}

Genetic studies were performed using frozen tissue samples in the collection of the Western Australian Museum (WAM) and the South Australian Museum (SAMA). Multiple samples from all key populations were included in order assess intraspecific geographic variation. Morphological examination was performed using formalin-fixed ethanol-preserved specimens in the WAM collection (Appendix 1).

\section{Allozyme analyses}

Analyses were undertaken on raw allozyme data generated previously for $D$. granariensis, $D$. 'NW Cape', D. mitchelli, and D. ornatus (Oliver et al. $2007 \mathrm{~b})$. All procedures relating to the use of stepwise Principle Co-ordinates Analysis (PCoA) and the estimation of genetic distances follow those detailed therein. Genetic distance matrices for both percent fixed differences (\%FD) and Nei's Distance (Nei D) were used to explore the phenetic and phylo-genetic affinities among taxa, the former via UPGMA dendrograms and the latter via NJ networks. Horner and Adams (2007) provide details of how dendrograms and trees were constructed. The level of statistical support for nodes was assessed by non-parametric bootstrapping for 1,000 pseudo-replicates using BASIC computer programs written by M. Adams.

\section{Mitochondrial DNA}

Mitochondrial DNA data were obtained from a subset of the animals used in the allozyme analyses. This sampling included all allozyme localities for nominal $D$. mitchelli and most localities for $D$. granariensis. These data were used to provide an independent assessment of genetic divergence and evolutionary relationships. The final mitochondrial dataset was comprised of 11 nominal $D$. granariensis, $4 \mathrm{D}$. mitchelli and 3 specimens of the
Northwest Cape population. Two specimens of three different species were included as outgroups based on the analyses of Oliver et al. (2007b): D. ornatus, $D$. pulcher and an undescribed Diplodactylus from the southern coast of Australia. The majority of sequences included in this study have already been published in Oliver et al. (2007b).

DNA extraction and amplification protocols for the ND2 gene follow those outlined in Oliver et al. (2007a,b). An approximately 900-bp segment of coding region was amplified using the following primers; light strand 5'-GCC CAT ACC CCG AAA ATS TTG-3' (modified from Jennings et al. 2003) and heavy 5'-TTA GGG TRG TTA TTT GHG AYA TKC G-3' (Oliver et al. 2007b). This fragment of the ND2 gene has been successfully used to examine intrageneric relationships in many diplodactyline gecko groups (Jennings et al. 2003; Melville et al. 2004; Pepper et al. 2006; Bauer et al. 2006; Oliver et al. 2007a,b). PCR products were amplified using Hotmaster Taq (Eppendorf) using the following conditions: denaturing $94^{\circ} \mathrm{C}$, annealling $55^{\circ} \mathrm{C}$ and for extension $72^{\circ} \mathrm{C}$ for 34 amplification cycles. Newly obtained PCR products for this study were sequenced in both directions by the company Macrogen of South Korea. New sequences were aligned with a pre-existing dataset from Oliver et al. (2007b) using Se-al (version 1.0 1 1: Rambaut, 1996).

Maximum Parsimony (MP) analyses were performed using PAUP (Version 4: Swofford 2000). We used the heuristic search algorithm with 100 random additions to trees. A bootstrap analysis with 500 heuristic pseudoreplicates was carried out to assess the robustness nodes in the MP tree. Bayesian analyses were performed using MrBayes V3.0 (Ronquist and Huelsenbeck 2003). A model of molecular evolution appropriate to our data was determined using MrModeltest (Nylander 2004). Monte Carlo Markov Chain sampling was run for $1,000,000$ generations sampling every 100 generations. The burnin was set at 100,000 generations after plotting generation number against likelihood scores indicated that stationarity had been reached well inside this point.

\section{Morphometrics}

Table 1 lists the characters and their abbreviations we measured for all species in the analysis. We also calculated the following ratios: TrunkL/SVL, HeadL/SVL, HeadW/SVL, HeadH/SVL, ArmL/SVL and LegL/SVL and also TailL as a percentage of SVL (TailL\%SVL). As body size differed among taxa and sex (see Results), we carried out 2-way ANCOVAs on TrunkL, ArmL, LegL, HeadL, HeadW, HeadH, NolO and NoSC with SVL as a covariate. In addition, we obtained radiographs of four individuals of $D$. mitchelli from the Pilbara (WAM R14823, R84458, R113618, R113642) and the 
Table 1 Meristic characters and their abbreviations used in this study.

\begin{tabular}{|c|c|}
\hline SVL & Snout-vent length \\
\hline $\mathrm{TL}$ & Tail length \\
\hline TrunkL & Trunk length from axilla to groin \\
\hline TailW & Tail width at widest point on original tails \\
\hline ArmL & Foreleg length from elbow to tip of $4^{\text {th }}$ finger \\
\hline LegL & Tibia length from upper surface of knee to tip of $4^{\text {th }}$ toe \\
\hline HeadL & Head length from tip of snout to retroarticular process \\
\hline HeadW & Head width at widest point \\
\hline HeadH & Head height at highest point \\
\hline OrbL & Orbit length - maximum \\
\hline NarEye & Distance from nare to anterior corner of eye \\
\hline SnEye & Distance from snout to anterior corner of eye \\
\hline EyeEar & Distance from posterior edge of eye to anterior margin of ear \\
\hline INar & Internarial distance \\
\hline $\mathrm{IO}$ & Interorbital width at centre of eyes \\
\hline SupLab & Number of supralabial scales \\
\hline Inflab & Number of infralabial scales \\
\hline Rcrease? & Whether the rostral was smooth or creased at all \\
\hline Creasel & Proportional length of the crease \\
\hline IntNar & Number of internarial scales \\
\hline $\mathrm{NolO}$ & Number of scales between center of orbits \\
\hline NoSC & Number of subcaudal scales from fracture plane to tip \\
\hline PostNas & Number of postnasal scales \\
\hline Rellab & Relative height of $1^{\text {st }}$ and $2^{\text {nd }}$ labial scales: $1-1^{\text {st }}>2^{\text {nd }} ; 2-1^{\text {st }}>=2^{\text {nd }} ; 3-1^{\text {st }}=2^{\text {nd }} ; 4-1^{\text {st }}<=2^{\text {nd }} ; 5-1^{\text {st }}<2^{\text {nd }}$ \\
\hline CSpurs & Average number of enlarged cloacal spurs \\
\hline $4 \mathrm{FL}$ & $4^{\text {th }}$ finger length from base to tip \\
\hline $4 \mathrm{FW}$ & $4^{\text {th }}$ finger width near distal joint \\
\hline 4FscanW & Width of scansor of $4^{\text {th }}$ finger \\
\hline 4FLam 1 & Number of undivided lamellae on $4^{\text {th }}$ finger \\
\hline 4FLam2 & Total number of lamellae on $4^{\text {th }}$ finger \\
\hline $4 \mathrm{TL}$ & $4^{\text {th }}$ toe length from base to tip \\
\hline $4 \mathrm{TW}$ & $4^{\text {th }}$ toe width near distal joint \\
\hline 4 TscanW & Width of scansor of $4^{\text {th }}$ toe \\
\hline 4TLam1 & Number of undivided lamellae on $4^{\text {th }}$ toe \\
\hline 4TLam2 & Total number of lamellae on $4^{\text {th }}$ toe \\
\hline MenL/W & Ratio of length/width of mental scale \\
\hline
\end{tabular}

Northwest Cape (WAM R81328, R102843, R132466, R132467) for vertebrae counts.

\section{RESULTS}

\section{Allozyme electrophoresis}

The final allozyme dataset comprised genotypes for 36 animals at 38 putative loci. An initial PCoA based on all specimens (Figure $2 \mathrm{~A}$ ) revealed the presence of three primary genetic groups, corresponding to $D$. ornatus, $D$. mitchelli, and the $D$. granariensis complex. The five $D$. 'NW Cape' specimens fell unequivocally into the latter group, demonstrating that their principal genetic affinities lie with $D$. granariensis rather than $D$. mitchelli. Follow-up PCoAs on all three primary genetic groups found no evidence for distinctive subgroups within $D$. ornatus or D. mitchelli (analyses not shown), but did reveal clearly defined subgroups within the D. granariensis complex (Figure2B). These subgroups correspond to 'NW Cape' (which was separated from true $D$. granariensis by the first dimension), and the two subspecies D. g. rex and $D$. g. granariensis. A final PCOA involving only individuals of $D$. granariensis (analysis not shown) provided additional support for the observation in Figure $2 \mathrm{~B}$ of a primary genetic dichotomy within $D$. granariensis which concords with the currentlyrecognized subspecies.

In summary, stepwise PCoA unreservedly placed each individual into one of five diagnosable units the four taxa already described plus the distinctive 'NW Cape' form. Allele frequencies at all polymorphic loci and pairwise genetic distances for these five taxa are presented in Tables 2 and 3, respectively. The three described species are diagnosable from one another by a minimum of 
Table 2 Allozyme frequencies for the five taxa under investigation. For polymorphic loci, the frequencies of all but the rarer/rarest alleles are expressed as percentages and shown as superscripts (allowing the frequency of each rare allele to be calculated by subtraction from $100 \%$ ). Alleles joined without being separated by a comma all shared the frequency indicated. A dash indicates no genotypes assignable at this locus. Taxon codes as per Figure 1. The maximum number of haploid genomes sampled for each taxon in shown in brackets. Invariant loci: $A k, M d h, N d p k$, and Srdh.

\begin{tabular}{|c|c|c|c|c|c|}
\hline Locus & $\begin{array}{l}\text { ORN } \\
(8)\end{array}$ & $\begin{array}{c}\text { MITCH } \\
\text { (14) }\end{array}$ & $\begin{array}{c}\text { CAPE } \\
(10)\end{array}$ & $\begin{array}{l}\text { REX } \\
(20)\end{array}$ & $\begin{array}{c}\text { GRAN } \\
\text { (20) }\end{array}$ \\
\hline Acon-1 & $a$ & $a^{57}, d$ & d & $d^{40}, b^{15}, f$ & $b^{71}, a^{15}, c d e "$ \\
\hline Acon-2 & $a^{75}, b^{13}, d$ & $b^{2}, d$ & d & $d^{35}, c^{35}, e$ & $e^{+7}, d^{2}, b c g^{5}$ \\
\hline$A_{c p}$ & $a^{k 8}, b$ & a & $a$ & a & a \\
\hline Acyc & $c^{122}, a$ & a & $\mathrm{a}$ & $\mathrm{a}$ & $a^{4}, b$ \\
\hline Adh-2 & $b$ & $b$ & $b$ & $b^{(x)}, a$ & $b$ \\
\hline Dia & $c^{+x}, d$ & $a^{*, 1, c}, c$ & $\mathrm{a}$ & a & $a^{4}, b$ \\
\hline Enol & b & $b$ & $b$ & $b^{3}, a^{4}, c$ & $\mathrm{~b}$ \\
\hline Est & $b^{*,}, a$ & $\mathrm{~d}^{56,}, \mathrm{c}$ & c & c & $c^{(x)}, \mathrm{b}$ \\
\hline$F d p$ & b & $b^{4,3}, a$ & b & $b$ & $\mathrm{~b}$ \\
\hline Fum & $b$ & $b^{\text {iti }}, a$ & b & $b^{(i)}, a$ & $b^{911}, a$ \\
\hline Gopd & a & $b$ & b & $b^{45}, a^{15}$ & b \\
\hline Gda & $b^{x}, a$ & a & a & a & a \\
\hline Glo & $b^{s, a}$ & b & b & $b$ & $b$ \\
\hline Got-1 & $b^{x}, a$ & $b$ & $b$ & $b$ & $\mathrm{~b}$ \\
\hline Got-2 & $\mathrm{b}$ & b & $a^{711}, b$ & $b^{4 \cdot}, c$ & $b$ \\
\hline Gpi & $b^{n+c}, c$ & b & b & b & $b^{0,5}, a$ \\
\hline Guk & $b^{\prime}$ & b & $d^{911}, b$ & $\mathrm{~b}$ & $b^{x / 2}, a^{5}, c$ \\
\hline$H b d h$ & - & a & $\mathrm{b}$ & $c^{(x)}, b^{4 i)}$ & $b^{961}, d^{\prime \prime \prime}$ \\
\hline Idh-1 & $\mathrm{b}^{\mathrm{W}}, \mathrm{a}$ & b & b & b & $\mathrm{b}$ \\
\hline$l d h-2$ & a & $\mathrm{b}$ & $a^{0}, b$ & $a^{a}, b$ & $a^{65}, b$ \\
\hline Lap & $c^{b 3}, b^{l i}, d$ & c & $\mathrm{c}^{\mathrm{br}}, \mathrm{a}$ & c & $c^{4}, b$ \\
\hline Ldh-1 & $b$ & $b$ & $b$ & $b^{97}, a$ & $b$ \\
\hline$L d h-2$ & $b$ & a & a & $a$ & a \\
\hline$M \mathrm{e}-1$ & $b^{-5}, d$ & c & $b^{511}, d^{+10}, e$ & $\mathrm{~b}^{3}, \mathrm{a}^{15}, \mathrm{~d}$ & $b^{6 n, 7}, d$ \\
\hline $\mathrm{Me}-2$ & $\mathrm{c}^{5}, \mathrm{a}$ & c & b & $\mathrm{cd}^{+5}, \mathrm{ab}^{\overline{1}}$ & $c^{35}, d^{25}, a b^{2 !}$ \\
\hline$M p i$ & $\mathrm{~b}$ & $\mathrm{~b}$ & b & $b^{+(1)}, a c^{7}$ & $b^{45}, a$ \\
\hline PepA-1 & $b$ & a & c & $c^{\mathrm{a}_{1}}, \mathrm{ad}^{5}$ & $a^{a i}, c$ \\
\hline$P$ epB & e & $\mathrm{d}$ & d & $\mathrm{h}^{10}, \mathrm{~d}^{m,}, \mathrm{a}$ & $f^{31}, b^{25}, d g^{15}, c^{14}, a$ \\
\hline Pgam & $\mathrm{a}$ & $b^{93}, a$ & $\mathrm{~b}$ & $b^{9 ! 1}, a$ & $a, b$ \\
\hline $6 P g d$ & $c^{-5}, \mathrm{e}$ & $\mathrm{a}$ & c & $c^{* 1}, \mathrm{~d}$ & $c^{91}, b d^{5}$ \\
\hline$P g_{m-1}$ & $\mathrm{f}^{51}, \mathrm{~g}^{25}, \mathrm{~b}^{13}, \mathrm{~d}$ & $g^{79}, h^{14}, f$ & $f^{\infty}, \mathrm{d}$ & $\mathrm{F}^{5}, \mathrm{~d}^{5}, \mathrm{ag}^{5}$ & $\mathrm{f}^{+5}, \mathrm{~b}^{211}, \mathrm{eg}^{\prime \prime \prime}, \mathrm{acd}^{5}$ \\
\hline$P g m-2$ & a & $\mathrm{b}$ & $\mathrm{b}$ & $\mathrm{b}$ & $b^{9}, c$ \\
\hline Sod & $a^{a}, c$ & $\mathrm{~b}$ & a & a & $a$ \\
\hline$T p i$ & $\mathrm{~b}$ & a & a & a & $a$ \\
\hline
\end{tabular}

Table 3 Pairwise genetic distances among the five taxa. Lower triangle $=\% \mathrm{FD}$ (allowing a $10 \%$ tolerance for shared alleles, see Oliver et al. [2007]); upper triangle $=$ Nei Ds.

\begin{tabular}{lccccc}
\hline Taxon & ORN & MITCH & CAPE & REX & GRAN \\
\hline ORN & - & 0.53 & 0.54 & 0.43 & 0.36 \\
MITCH & 32 & - & 0.32 & 0.26 & 0.21 \\
CAPRE & 32 & 24 & - & 0.11 & 0.12 \\
REX & 24 & 16 & 5 & - & 0.07 \\
GRAN & 22 & 11 & 5 & 0 & - \\
\hline
\end{tabular}

four and a maximum of 12 fixed differences (range 11-32\%FD, Table 2), whereas the two subspecies of D. granariensis displayed no fixed differences between each other and only a modest Nei D of 0.07. The 'NW Cape' taxon, while clearly allied genetically with D. granariensis to the exclusion of
D. mitchelli, nevertheless displays two fixed differences $(5 \% \mathrm{FD}$, Table 3 ) involving three loci (Acon-1, Guk and Me-2; Table 2) when compared to both D. g. rex and D.g. granariensis. A further three loci display major differences in allele frequency ( $p$ $>50 \%$ for Acon-2, Got-2, and PepB; Table 2) between 'NW Cape' and both subspecies of $D$. granariensis.

Figure 3 contrasts the overall genetic similarities among the five taxa (as assessed by UPGMA analyses) with their comparative phylogenetic affinities, as assessed using the NJ approach). Although the 'NW Cape' taxon is genetically less similar to the two subspecies of $D$. granariensis than either is from the other (Figure 2B), the phylogenetic analyses suggest that its genetic affinities lie within D. granariensis in general and in particular with the geographically more proximate northern subspecies of D. g. rex. 


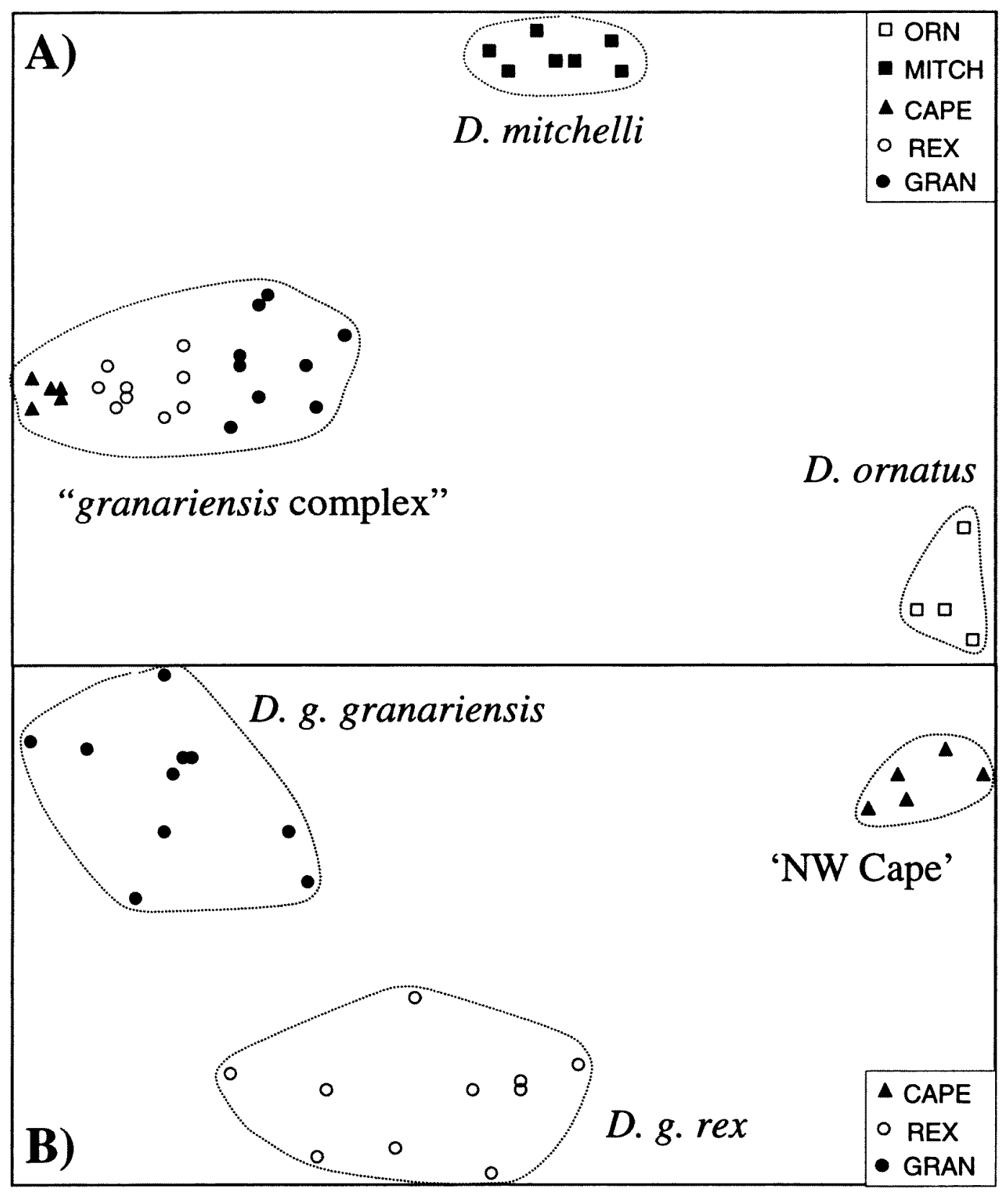

Figure 2 Summary of Stepwise PCoA of western Diplodactylus, demonstrating how individuals were assigned to taxa on genetic criteria. A) Initial PCoA on all 36 individuals. The relative PCoA scores have been plotted for the first (X-axis) and second (Y-axis) dimensions, which individually explained $24 \%$ and $22 \%$ respectively of the total multivariate variation. B) Follow-up PCoA on the 25 individuals comprising the $D$. granariensis complex of A). The first and second dimensions individually explained $22 \%$ and $17 \%$ respectively of the total multivariate variation. Taxon code: $\mathrm{ORN}=D$. ornatus, $\mathrm{MITCH}=D$. mitchelli, $\mathrm{CAPE}={ }^{\mathrm{N} W}$ Cape', $\mathrm{REX}=D$. g. rex, GRAN = D. g. granariensis.

\section{Mitochondrial DNA}

The final aligned sequence comprised 749 base pairs from within the coding region of the ND2 gene. Of these sites, 474 were constant, 234 were variable and parsimony informative and 41 were variable and not parsimony informative. A single three base pair indel was present in all samples of D. mitchelli. MrModeltest indicated that the most appropriate model of molecular evolution for our dataset was GTR+I+G.

Both maximum parsimony and Bayesian analyses identified three major lineages within the Diplodactylus populations of interest; these correspond to $D$. mitchelli, the Northwest Cape population and both subspecies of $D$. granariensis (Figure 4). In all analyses these three lineages formed a strongly supported clade exclusive to all outgroups. Relationships among the three lineages were less clearly resolved. However there was a tendancy for $D$. mitchelli to be most divergent, and the Northwest Cape population and D. $g$. granariensis to be sister taxa. 
A) UPGMA

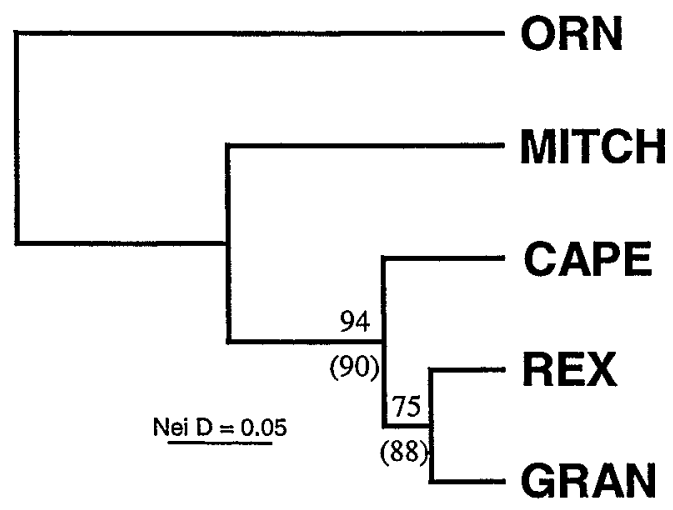

\section{B) $\mathbf{N J}$}

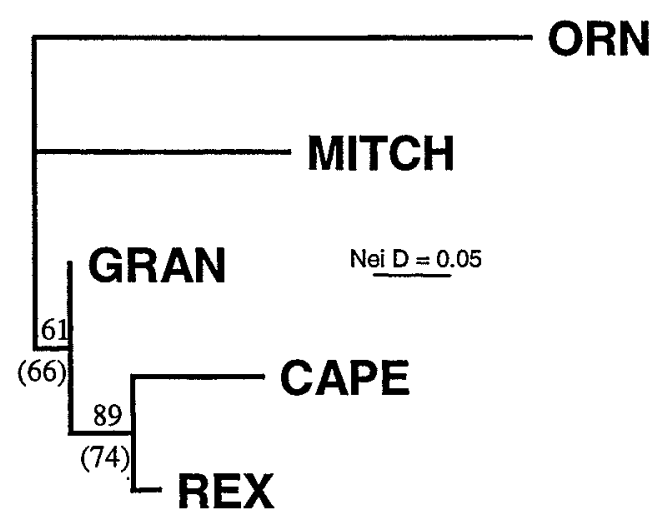

Figure 3 A comparison of the genetic and phylogenetic affinities of western Diplodactylus, based on Nei Distances. Analyses based on \%FD values (not presented) displayed the same arrangement of nodes. Taxon codes as per Figure 2. Bootstrap values above $60 \%$ are shown for all nodes (bracketed values below the branch are those obtained using \%FD). A) UPGMA dendrogram. B) NJ tree.

The Northwest Cape population showed very little intraclade genetic diversity, differing by a single change in one individual. Within $D$. mitchelli, one individual was notably divergent from the other three which were almost identical. Within D. granarienis, genetic divergences were discordant with the current subspecific taxonomy. This lineage was divided into two strongly supported clades, one is represented by only two animals from the western edge of the Darling Range near Perth (Bindoon), while the other occupies all of the species' distribution to the east of the Darling Range. Animals assigned to $D$. g. rex formed a very shallow clade nested within deeper lineages assigned to $D$. g. granariensis.

\section{Morphometrics}

Table 4 compares the four taxa for the morphological characters measured with the results of the statistical analyses. Sex had an effect on SVL, TrunkL, ArmL and LegL with females being significantly longer than males for these characters. There were no other sex differences for the other characters. However, SVL was a significant covariate for most of the morphological characters tested (Table 4). Diplodactylus mitchelli was the largest taxon, followed by $D$. g. rex, D. 'NW Cape' and $D . g$. granariensis. In contrast, D. g. granariensis had the relatively longest tail with the most supracaudal scales, followed by $D$. 'NW Cape', $D$. g. rex and $D$. mitchelli with a very short tail with fewer, larger scales. Limb length was also significantly different among species with both $D$. mitchelli and $D$. g. rex with longer arms and legs than D. 'NW Cape'and D. g. granariensis, although limb length as a proportion of SVL showed no strong trends. Diplodactylus $g$. rex had a much more massive head for all of the measures for head size, whereas $D$. mitchelli had an elongate head that was dorsoventrally compressed. The number of interorbital scales also varied, with $D$. mitchelli having fewer (owing to large scale size) and $D . g$. granariensis having much more numerous (and smaller) scales.

Some scale characters also showed differences among taxa. Rostral scales were almost always slightly creased in $D$. mitchelli and $D$. $g$. granariensis, but rarely so for $D$. 'NW Cape'and $D$. g. rex (reflected also in the length of the crease). Diplodactylus mitchelli was also notable for having a greater number of cloacal spurs and wider scansors than the other taxa. There were some differences with subdigital lamellae, with D. mitchelli and D. 'NW Cape' having fewer but with a higher proportion of undivided lamellae than both subspecies of $D$. granariensis. There were no differences among the four taxa in labial scales, mental scale ratios or other characters commonly used as diagnostic traits in geckos (Table 4). Vertebral counts for $D$. mitchelli from the Pilbara were all $26(\mathrm{~N}=4)$ whereas for the Northwest Cape population they were all $25(\mathrm{~N}=$ 4).

Colour and pattern of the four taxa also differed. The two subspecies of $D$. granariensis have a strong to slightly scalloped vertebral stripe, whereas both $D$. mitchelli and $D$. 'NW Cape' both have a more broken stripe with short tranverse bars. The ground colour of $D$. g. granariensis is a dark brown to almost black, D. g. rex is much paler and $D$. mitchelli and $D$. 'NW Cape' have a ground colour imbued with a rich red. 
Table 4 Summaries of characters and ratios measured for Diplodactylus. Mean \pm S.D. (range). Sample sizes are listed in column headings, unless noted for individual characters below. See Table 1 for abbreviations. SVL was tested with a 2-way ANOVA. 2-way ANCOVAs (factors - species and sex, covariate - SVL) were carried out (see text for explanation) and reported in the last column. All species $X$ sex interaction terms were not significant with alpha $=0.05$. Key: NS - not significant: $P>0.10$ $* \mathrm{P}<0.05, * * \mathrm{P}<0.01, * * * \mathrm{P}<0.001, * * * * \mathrm{P}<0.0001$

\begin{tabular}{|c|c|c|c|c|c|}
\hline Character & $\begin{array}{c}\text { D. 'Cape Range' } \\
\mathbf{N}=19\end{array}$ & $\begin{array}{l}\text { D. mitchelli } \\
\mathrm{N}=22\end{array}$ & $\begin{array}{l}\text { D. g. granariensis } \\
\mathrm{N}=32\end{array}$ & $\begin{array}{l}\text { D. g. rex } \\
\mathrm{N}=27\end{array}$ & Statistics \\
\hline SVL & $\begin{array}{c}\text { Female }(\mathrm{N}=9): \\
60.7 \pm 3.4 \\
(56.0-66.5) \\
\text { Males }(\mathrm{N}=10): \\
52.2 \pm 4.2 \\
(43.0-57.5)\end{array}$ & $\begin{array}{c}\text { Female }(\mathrm{N}=9) \text { : } \\
65.5 \pm 7.5 \\
(53.0-77.0) \\
\text { Males }(\mathrm{N}=13) \text { : } \\
63.3 \pm 5.7 \\
(47.5-71.5)\end{array}$ & $\begin{array}{c}\text { Female }(\mathrm{N}=12): \\
55.7 \pm 5.5 \\
(47.0-62.0) \\
\text { Males }(\mathrm{N}=20): \\
53.4 \pm 3.6 \\
(44.0-61.0)\end{array}$ & $\begin{array}{c}\text { Female }(\mathrm{N}=12) \text { : } \\
63.4 \pm 7.9 \\
(49.0-72.0) \\
\text { Males }(\mathrm{N}=15) \\
58.9 \pm 4.9 \\
(51.0-67.0)\end{array}$ & $\begin{array}{l}\text { Spp. }-F_{3,95}=16.834^{* * * *} \\
\text { Sex }-F_{1,95}=13.497^{* * * *}\end{array}$ \\
\hline TrunkL & $\begin{array}{c}\text { Female }(\mathrm{N}=7): \\
27.2 \pm 3.4 \\
(23.7-32.2) \\
\text { Males }(\mathrm{N}=12) \\
23.1 \pm 1.8 \\
(20.1-25.9)\end{array}$ & $\begin{array}{c}\text { Female }(\mathrm{N}=9): \\
31.3 \pm 4.3 \\
(23.4-38.4) \\
\text { Males }(\mathrm{N}=13) \text { : } \\
27.7 \pm 3.2 \\
(18.6-31.0)\end{array}$ & $\begin{array}{c}\text { Female }(\mathrm{N}=12): \\
25.0 \pm 2.8 \\
(29.0-39.0) \\
\text { Males }(\mathrm{N}=2): \\
22.7 \pm 2.9 \\
(16.9-28.7)\end{array}$ & $\begin{array}{c}\text { Female }(\mathrm{N}=12) \text { : } \\
28.4 \pm 3.7 \\
(20.8-32.5) \\
\text { Males }(\mathrm{N}=15) \text { : } \\
25.2 \pm 2.7 \\
(20.9-30.3)\end{array}$ & $\begin{array}{c}\text { Spp. }-F_{3,92}=1.704^{\mathrm{NS}} \\
\text { Sex }-\mathrm{F}_{1,92}=11.410^{* *} \\
\text { SVL }-\mathrm{F}_{1,92}=185.573^{* * *}\end{array}$ \\
\hline TailL & $\begin{array}{c}30.6 \pm 3.2 \\
(25.0-34.0) \\
N=8\end{array}$ & $\begin{array}{c}32.3 \pm 3.9 \\
(25.0-38.0) \\
\mathrm{N}=13\end{array}$ & $\begin{array}{c}33.0 \pm 3.7 \\
(22.0-39.0) \\
\mathrm{N}=29\end{array}$ & $\begin{array}{c}34.5 \pm 4.8 \\
(24.0-42.0) \\
N=20\end{array}$ & $\begin{aligned} \text { Spp. }-F_{3,60} & =15.699^{* * * *} \\
\text { Sex }-F_{1,60} & =1.756^{\mathrm{NS}} \\
\text { SVL }-F_{1,60} & =57.544^{* * * *}\end{aligned}$ \\
\hline ArmL & $\begin{array}{c}13.9 \pm 0.8 \\
(11.5-14.9) \\
N=18\end{array}$ & $\begin{array}{c}16.7 \pm 1.2 \\
(13.8-18.5) \\
N=21\end{array}$ & $\begin{array}{c}13.6 \pm 0.9 \\
(11.9-15.0) \\
N=31\end{array}$ & $\begin{array}{c}15.8 \pm 1.4 \\
(12.7-17.7)\end{array}$ & $\begin{array}{l}\text { Spp. }-F_{3,91}=24.043^{* * * *} \\
\text { Sex }-F_{1,91}=5.842^{*} \\
\text { SVL }-F_{1,91}=171.055^{* * *}\end{array}$ \\
\hline LegL & $\begin{array}{c}17.0 \pm 0.9 \\
(14.1-18.0) \\
N=16\end{array}$ & $\begin{array}{c}20.5 \pm 1.6 \\
(16.5-22.8) \\
N=21\end{array}$ & $\begin{array}{c}16.5 \pm 1.1 \\
(14.7-18.5) \\
N=30\end{array}$ & $\begin{array}{c}19.1 \pm 1.7 \\
(15.7-21.5) \\
N=26\end{array}$ & $\begin{array}{c}\text { Spp. }-\mathrm{F}_{3,87}=16.740^{* * * *} \\
\text { Sex }-\mathrm{F}_{1,87}=6.320^{*} \\
\text { SVL }-\mathrm{F}_{1,87}=91.514^{* * * *}\end{array}$ \\
\hline HeadL & $\begin{array}{c}15.7 \pm 1.2 \\
(12.2-17.3)\end{array}$ & $\begin{array}{c}17.2 \pm 1.3 \\
(14.1-19.3)\end{array}$ & $\begin{array}{c}15.0 \pm 0.8 \\
(13.3-16.4)\end{array}$ & $\begin{array}{c}17.3 \pm 1.7 \\
(14.3-20.0)\end{array}$ & $\begin{array}{c}\text { Spp. }-F_{3,94}=13.385^{* * *} \\
\text { Sex }-F_{1,94}=0.092^{\mathrm{NS}} \\
\text { SVL }-\mathrm{F}_{1,94}=337.651^{* * * *}\end{array}$ \\
\hline HeadW & $\begin{array}{c}10.5 \pm 1.0 \\
(8.3-12.3) \\
N=18\end{array}$ & $\begin{array}{c}11.7 \pm 1.1 \\
(9.0-13.4) \\
N=21\end{array}$ & $\begin{array}{c}10.7 \pm 0.8 \\
(8.9-12.3)\end{array}$ & $\begin{array}{c}12.7 \pm 1.4 \\
(10.0-15.7)\end{array}$ & $\begin{array}{c}\text { Spp. }-F_{3,92}=23.915^{* * * *} \\
\text { Sex }-F_{1,92}=2.890^{N S} \\
\text { SVL }-F_{1,92}=128.543^{* * * *}\end{array}$ \\
\hline HeadH & $\begin{array}{c}7.1 \pm 0.6 \\
(5.1-8.1) \\
N=18\end{array}$ & $\begin{array}{c}7.6 \pm 0.7 \\
(6.0-8.8) \\
N=21\end{array}$ & $\begin{array}{c}6.8 \pm 0.6 \\
(5.1-7.8)\end{array}$ & $\begin{array}{c}8.4 \pm 0.9 \\
(7.0-10.4)\end{array}$ & $\begin{aligned} \text { Spp. }-F_{3,92} & =21.324^{* * * *} \\
\text { Sex }-F_{1,92} & =0.579^{N S} \\
\text { SVL }-F_{1,92} & =55.967^{* * *}\end{aligned}$ \\
\hline
\end{tabular}


NoSC

$\mathrm{N}=11$

$(31-46)$
$\mathrm{N}=13$

$9.4 \pm 1.2$

$6.2 \pm 1.1$

(4.6-8.0)

6.7-11.3)

$\mathrm{N}=12$

$4.6 \pm 0.4$
$(3.4-5.2)$

$4.3 \pm 0.3$
$(3.7-4.9)$

$\mathrm{N}=18$

$5.0 \pm 0.4$

$4.4 \pm 0.4$

(3.7-5.0)

(4.2-5.5)

$6.8 \pm 0.5$

(5.9-7.9)

$6.1 \pm 0.5$
$(4.9-6.8)$

$\mathrm{N}=18$

EyeEar

$5.9 \pm 0.6$

(4.6-6.8)

$\mathrm{N}=18$

$6.3 \pm 0.8$

(4.7-8.3)

$1.9 \pm 0.1$

(1.6-2.1)

IO

$5.3 \pm 0.5$

$(4.1-6.0)$
$N=18$

$2.1 \pm 0.2$

(1.8-2.3)

$5.9 \pm 0.4$

(5.1-6.5)

$11.0 \pm 0.7$

SupLab

(10-12)

$11.4 \pm 0.8$

(10-13)

$11.3 \pm 0.9$

(10-13)

$10 / 10$

$0.25 \pm 0.16$

$(0-0.5)$

$(63-84)$
$N=25$

$60 \pm 3$

$(55-67)$
$N=20$

$6.4 \pm 1.0$

(4.6-8.0)

$\mathrm{N}=10$

$7.6 \pm 1.0$

(5.8-9.5)

$\mathrm{N}=21$

$4.2 \pm 0.4$

(3.6-4.9)

$4.5+0.5$

$\mathrm{N}=31$

$4.4 \pm 0.4$

(3.9-5.6)

(3.9-6.5)

$\begin{aligned} \text { Spp. }-F_{3,92} & =41.241^{* * *+} \\ \text { Sex }-F^{* 92} & =0.200^{\mathrm{NS}}\end{aligned}$ $\mathrm{SVL}-\mathrm{F}_{1,92}=0.152^{\mathrm{NS}}$

Spp. $-\mathrm{F}_{3,62}=128.128^{* * * *}$

Sex $-F_{1,62}=0.399^{\mathrm{NS}}$
$\mathrm{SVL}-\mathrm{F}_{1,62}=0.465^{\mathrm{NS}}$

$5.9 \pm 0.4$

(5.0-6.6)

$6.6 \pm 0.6$

(5.3-7.9)

$6.4 \pm 0.8$

(5.0-8.0)

(4.5-6.2)

$1.9 \pm 0.1$

(1.7-2.1)

$5.4 \pm 0.4$

$(4.7-6.2)$
$N=27$

$119 \pm 0.9$

$11.9 \pm 0.9$
$(10-15)$

$11.9 \pm 0.9$

(10-15)

$32 / 32$

$0.32 \pm 0.10$

(0.125-0.5) 
Table 4 (cont.)

\begin{tabular}{|c|c|c|c|c|c|}
\hline Character & $\begin{array}{c}D . \text { 'Cape Range' } \\
\mathbf{N}=19\end{array}$ & $\begin{array}{l}\text { D. mitchelli } \\
\mathrm{N}=22\end{array}$ & $\begin{array}{l}D . g \cdot \text { granariensis } \\
\mathbf{N}=32\end{array}$ & $\begin{array}{l}\text { D. g. rex } \\
\mathrm{N}=27\end{array}$ & Statistics \\
\hline IntNar & $3 / 19$ & $6 / 22$ & $4 / 32$ & $3 / 27$ & \\
\hline PostNas & $\begin{array}{c}2.82 \pm 0.51 \\
\quad(2-4)\end{array}$ & $\begin{array}{c}2.48 \pm 0.50 \\
(2-3)\end{array}$ & $\begin{array}{c}3.05 \pm 0.32 \\
\quad(2-3)\end{array}$ & $\begin{array}{c}3.06 \pm 0.63 \\
(1-4)\end{array}$ & \\
\hline RelLab & $\begin{array}{l}4.26 \pm 0.87 \\
(3-5)\end{array}$ & $\begin{array}{c}3.05 \pm 1.46 \\
(1-5)\end{array}$ & $\begin{array}{c}3.44 \pm 1.01 \\
(1-5)\end{array}$ & $\begin{array}{c}2.89 \pm 1.05 \\
(1-5)\end{array}$ & \\
\hline CSpurs & $\begin{array}{c}5.2 \pm 0.4 \\
(5-6) \\
N=10\end{array}$ & $\begin{array}{c}7.3 \pm 1.0 \\
(5.5-9.5) \\
N=13\end{array}$ & $\begin{array}{c}5.3 \pm 0.9 \\
(3-7) \\
N=20\end{array}$ & $\begin{array}{c}5.1 \pm 1.3 \\
(3.5-8.5) \\
N=15\end{array}$ & \\
\hline $4 \mathrm{FL}$ & $\begin{array}{c}3.8 \pm 0.2 \\
(3.6-4.1) \\
N=13\end{array}$ & $\begin{array}{c}4.5 \pm 0.5 \\
(3.6-5.3) \\
N=20\end{array}$ & $\begin{array}{c}3.6 \pm 0.3 \\
(3.1-4.2) \\
\mathrm{N}=24\end{array}$ & $\begin{array}{c}4.1 \pm 0.4 \\
(3.4-4.7) \\
N=16\end{array}$ & \\
\hline $4 \mathrm{FW}$ & $\begin{array}{c}0.7 \pm 0.1 \\
(0.5-0.9) \\
N=13\end{array}$ & $\begin{array}{c}0.9 \pm 0.1 \\
(0.7-1.1) \\
N=21\end{array}$ & $\begin{array}{c}0.7 \pm 0.1 \\
(0.6-0.9) \\
N=25\end{array}$ & $\begin{array}{c}0.8 \pm 0.1 \\
(0.6-0.9) \\
N=18\end{array}$ & \\
\hline 4FscanW & $\begin{array}{c}1.2 \pm 0.1 \\
(1.1-1.4) \\
N=12\end{array}$ & $\begin{array}{c}1.5 \pm 0.2 \\
(1.1-1.8) \\
N=21\end{array}$ & $\begin{array}{c}1.1 \pm 0.1 \\
(0.9-1.3) \\
N=25\end{array}$ & $\begin{array}{c}1.1 \pm 0.1 \\
(0.8-1.3) \\
N=17\end{array}$ & \\
\hline 4FLam1 & $\begin{array}{c}6.1 \pm 0.3 \\
(6-7) \\
N=13\end{array}$ & $\begin{array}{c}6.0 \pm 0.5 \\
(5-7) \\
N=21\end{array}$ & $\begin{array}{c}4.9 \pm 1.2 \\
(3-7) \\
N=27\end{array}$ & $\begin{array}{c}4.6 \pm 0.8 \\
(4-6) \\
N=19\end{array}$ & \\
\hline 4FLam2 & $\begin{array}{c}6.2 \pm 0.4 \\
(6-7) \\
N=13\end{array}$ & $\begin{array}{c}6.5 \pm 0.6 \\
(5-7) \\
N=21\end{array}$ & $\begin{array}{c}7.0 \pm 0.7 \\
(6-8) \\
\mathrm{N}=27\end{array}$ & $\begin{array}{c}7.2 \pm 0.6 \\
(6-8) \\
N=19\end{array}$ & \\
\hline 4TL & $\begin{array}{c}4.3 \pm 0.1 \\
(4.1-4.4) \\
N=9\end{array}$ & $\begin{array}{c}5.3 \pm 0.5 \\
(4.5-6.2) \\
N=19\end{array}$ & $\begin{array}{c}4.4 \pm 0.3 \\
(3.9-5.0) \\
N=20\end{array}$ & $\begin{array}{c}4.9 \pm 0.5 \\
(4.1-5.5) \\
N=11\end{array}$ & \\
\hline $4 \mathrm{TW}$ & $\begin{array}{c}0.8 \pm 0.1 \\
(0.6-0.9) \\
\mathrm{N}=11\end{array}$ & $\begin{array}{c}0.9 \pm 0.1 \\
(0.7-1.2) \\
\mathrm{N}=21\end{array}$ & $\begin{array}{c}0.8 \pm 0.1 \\
(0.6-0.9) \\
N=22\end{array}$ & $\begin{array}{c}0.9 \pm 0.1 \\
(0.6-1.1) \\
N=16\end{array}$ & \\
\hline
\end{tabular}




\begin{tabular}{|c|c|c|c|c|}
\hline 4TscanW & $\begin{array}{c}1.4 \pm 0.1 \\
(1.2-1.6) \\
N=11\end{array}$ & $\begin{array}{c}1.7 \pm 0.2 \\
(1.3-2.0) \\
\mathrm{N}=21\end{array}$ & $\begin{array}{c}1.2 \pm 0.2 \\
(1.0-1.4) \\
\mathrm{N}=22\end{array}$ & $\begin{array}{c}1.3 \pm 0.2 \\
(0.9-1.6) \\
\mathrm{N}=18\end{array}$ \\
\hline 4TLam1 & $\begin{array}{c}7.2 \pm 0.9 \\
(6-9) \\
N=13\end{array}$ & $\begin{array}{c}6.8 \pm 1.5 \\
(3-8) \\
N=21\end{array}$ & $\begin{array}{c}5.0 \pm 1.5 \\
(3-9) \\
N=27\end{array}$ & $\begin{array}{c}5.2 \pm 1.0 \\
(3-8) \\
\mathrm{N}=18\end{array}$ \\
\hline 4TLam2 & $\begin{array}{c}7.9 \pm 0.7 \\
(7-9) \\
\mathrm{N}=13\end{array}$ & $\begin{array}{l}7.9 \pm 0.6 \\
(7-9) \\
N=21\end{array}$ & $\begin{array}{l}8.7 \pm 0.9 \\
(7-11) \\
N=27\end{array}$ & $\begin{array}{c}8.9 \pm 0.6 \\
(8-10) \\
N=18\end{array}$ \\
\hline MenL/W & $\begin{array}{l}1.2 \pm 0.1 \\
(1.1-1.4)\end{array}$ & $\begin{array}{c}1.3 \pm 0.2 \\
(1.1-1.7) \\
N=10\end{array}$ & $\begin{array}{c}1.1 \pm 0.2 \\
(0.8-1.8) \\
N=31\end{array}$ & $\begin{array}{c}1.3 \pm 0.2 \\
(0.9-1.8)\end{array}$ \\
\hline TrunkL/SVL & $\begin{array}{c}0.44 \pm 0.03 \\
(0.40-0.49) \\
N=17\end{array}$ & $\begin{array}{c}0.45 \pm 0.03 \\
(0.39-0.53)\end{array}$ & $\begin{array}{c}0.43 \pm 0.04 \\
(0.36-0.52)\end{array}$ & $\begin{array}{c}0.44 \pm 0.03 \\
(0.37-0.50)\end{array}$ \\
\hline HeadL/SVL & $\begin{array}{c}0.28 \pm 0.01 \\
(0.26-0.30) \\
N=19\end{array}$ & $\begin{array}{c}0.27 \pm 0.03 \\
(0.25-0.30)\end{array}$ & $\begin{array}{c}0.28 \pm 0.02 \\
(0.25-0.32)\end{array}$ & $\begin{array}{c}0.28 \pm 0.01 \\
(0.26-0.31)\end{array}$ \\
\hline HeadW/SVL & $\begin{array}{c}0.19 \pm 0.01 \\
(0.16-0.20) \\
N=18\end{array}$ & $\begin{array}{c}0.18 \pm 0.01 \\
(0.16-0.19) \\
N=21\end{array}$ & $\begin{array}{c}0.20 \pm 0.01 \\
(0.17-0.23)\end{array}$ & $\begin{array}{c}0.21 \pm 0.02 \\
(0.18-0.24)\end{array}$ \\
\hline HeadH/SVL & $\begin{array}{c}0.13 \pm 0.01 \\
(0.11-0.14) \\
N=18\end{array}$ & $\begin{array}{c}0.12 \pm 0.01 \\
(0.10-0.14) \\
N=21\end{array}$ & $\begin{array}{c}0.13 \pm 0.01 \\
(0.09-0.16)\end{array}$ & $\begin{array}{c}0.14 \pm 0.01 \\
(0.11-0.16)\end{array}$ \\
\hline ArmL/SVL & $\begin{array}{c}0.25 \pm 0.02 \\
(0.22-0.29) \\
N=18\end{array}$ & $\begin{array}{c}0.26 \pm 0.02 \\
(0.25-0.30) \\
N=21\end{array}$ & $\begin{array}{c}0.25 \pm 0.02 \\
(0.23-0.32) \\
\mathrm{N}=31\end{array}$ & $\begin{array}{c}0.26 \pm 0.01 \\
(0.24-0.29)\end{array}$ \\
\hline LegL/SVL & $\begin{array}{c}0.30 \pm 0.02 \\
(0.26-0.33) \\
N=16\end{array}$ & $\begin{array}{c}0.32 \pm 0.02 \\
(0.28-0.36) \\
N=21\end{array}$ & $\begin{array}{c}0.31 \pm 0.03 \\
(0.27-0.38) \\
\mathrm{N}=30\end{array}$ & $\begin{array}{c}0.31 \pm 0.02 \\
(0.27-0.36) \\
N=26\end{array}$ \\
\hline Tail\%SVL & $\begin{array}{c}56.6 \pm 2.2 \\
(52.4-60.0) \\
N=8\end{array}$ & $\begin{array}{c}49.4 \pm 3.9 \\
(43.9-56.7) \\
N=13\end{array}$ & $\begin{array}{c}63.9 \pm 4.4 \\
(55.7-71.0) \\
N=24\end{array}$ & $\begin{array}{c}58.1 \pm 6.1 \\
(45.2-70.2) \\
N=21\end{array}$ \\
\hline
\end{tabular}




\section{DISCUSSION}

Our molecular and morphological results were not always consistent with current taxonomic arrangements, specifically not strongly supporting the subspecies within $D$. granariensis and supporting the Northwest Cape taxon as distinct from $D$. mitchelli and $D$. granariensis. We review all of the evidence below to arrive at our taxonomic conclusions.

Despite consistent morphological differences and allozyme profiles, there was only weak support for $D$. g. rex as a valid subspecies within D. granariensis with mtDNA data which indicated very recent divergence. Neither molecular dataset provided diagnostic markers which could unequivocally identify animals as belonging to one or the other subspecies or a hybrid between the two. Moreover, the two different molecular techniques presented a different picture of where the most significant phylogeographic split occurs, with allozymes indicating a split to distinguish northern versus southern populations (i.e., generally concordant with the subspecific taxonomy; Figure 2B), whereas mtDNA placed it across either side of the Darling Range (Figure 4), with a further divide separating wheatbelt $D$. g. granariensis from northern $D$. g. rex. Although the allozyme data mirrored the morphological differences, the mtDNA patterns indicated that $D$. g. rex populations were more closely related to $D$. g. granariensis from the wheatbelt; furthermore, the Darling Range (near Perth) population of $D . g$.

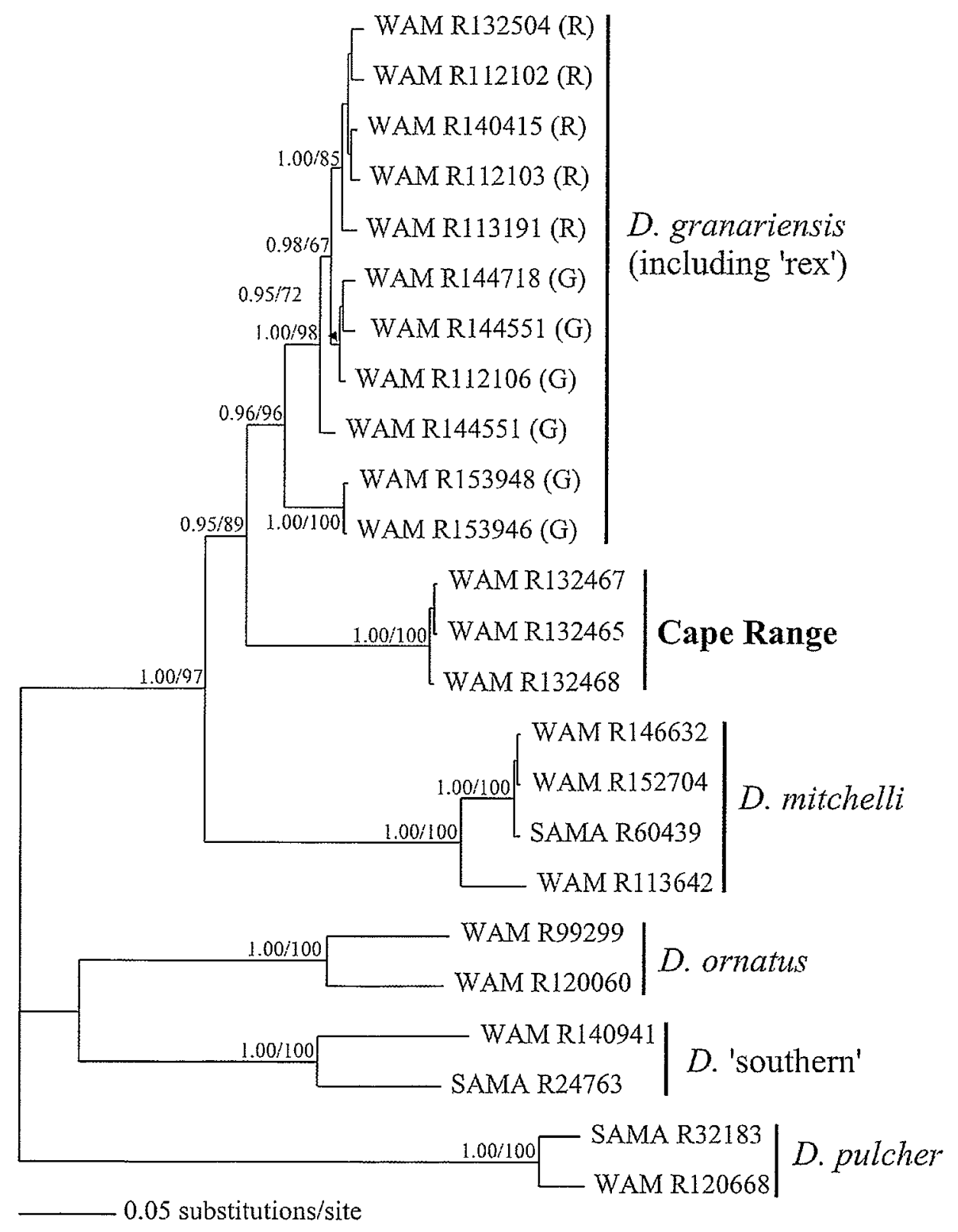

Figure 4 Bayesian consensus tree of inferred phylogenetic relationships of northwestern Diplodactylus based on 749 bp of the mitochondrial ND2 gene. Posterior probabilities above $95 \%$ and bootstrap support values above $50 \%$ are shown for major nodes. 
granariensis formed the sister taxon to wheatbelt $D$. g. granariensis and D. g. rex. All branches were very shallow relative to other species in our analysis.

The subspecies D. g. rex was named by Storr (1988) based on a combination of unusual characters possessed by the populations to the north of typical D. g. granariensis (Figure 1). These characters include large body size, uncreased rostral scale and more bold vertebral stripe with little pattern on the sides. The relatively low level of $m+D N A$ differentiation between populations of D. g. rex and D. g. granariensis contrasts strikingly with significant morphological differentiation. Further work on this interesting problem is continuing, as this may be an instance of active speciation. The evolution of the massive head in D. g. rex suggests that the maximum prey size that is able to be taken may be a factor, although other hypotheses such as phenotypic plasticity, allometry and character displacement could play a role.

Our second finding was that the Northwest Cape population of $D$. 'mitchelli' was distinct from $D$. mitchelli from the Pilbara. The genetic data strongly supported a closer relationship with $D$. granariensis than with $D$. mitchelli, although the morphological data was ambiguous as to their affinities. We describe the Northwest Cape population as a new species below.

\section{SYSTEMATICS}

\section{Reptilia}

Squamata

Family Diplodactylidae Underwood, 1954

\section{Genus Diplodactylus Gray, 1832}

\section{Type species}

Diplodactylus vittatus Gray, 1832, by monotypy.

\section{Diagnosis}

A genus of Diplodactylidae (sensu Han et al. 2004) characterized by robust habitus, wide scansors, short $(<80 \%$ SVL) stout tails, absence of preanal pores, numerous ( $>$ 5) clocal spurs, two pairs of cloacal bones and anteriorly enlarged jugal bone entering floor of lacrimal foramen (Oliver et al. 2007a).

\section{Diplodactylus capensis sp. nov. Cape Range Stone Gecko Figures 5, 6}

\section{Material examined}

\section{Holotype}

WAM R154901 in the collection of the Western
Australian Museum, Perth. An adult male collected at Shothole Canyon, Northwest Cape, Western Australia $\left(22^{\circ} 03^{\prime} 12^{\prime \prime} S, 114^{\circ} 01^{\prime} 11^{\prime \prime E}\right)$ by P. Doughty on 13 April 2004. Liver sample stored at $-75^{\circ} \mathrm{C}$ at WAM.

\section{Paratypes}

WAM R81328 (F) $6 \mathrm{~km} \mathrm{~S}$ of Exmouth (21 58'S, 114.06'E); WAM R81342 (M) Shothole Canyon $\left(22^{\circ} 05^{\prime} \mathrm{S}, 114^{\circ} 02^{\prime} \mathrm{E}\right)$; WAM R102843 (M) Cape Range N.P. (2204'17"S, $\left.114^{\circ} 00^{\prime} 23^{\prime \prime} \mathrm{E}\right) ;$ WAM R102900 (F) Cape Range N.P. (2200901"S, $\left.113^{\circ} 59^{\prime} 52^{\prime \prime E}\right)$; WAM R117870 (F) Shothole Canyon (22 ${ }^{\circ} 2^{\prime} S$, 114 $02^{\prime} \mathrm{E}$ ); WAM R132465 and WAM R132466 (both F)

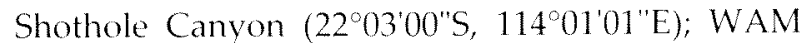
R141700 (M) Learmonth (22.14'31'S, 11402'02"E); WAM R154902 (M) $9 \mathrm{~km} \mathrm{~S}$ of Exmouth (22.01'18"S, $\left.114^{\circ} 06^{\prime} 39^{\prime \prime} \mathrm{E}\right)$.

\section{Diagnosis}

A medium-large member of Diplodactylus characterised by stout head with tall labial scales, mental similar size to infralabials, elongate tail, wide scansors with a row of discrete unbroken lamellae along digit, medium-sized dorsal scales, 25 vertebrae and by light reddish brown dorsal colouration with pale continuous dorsal stripe with transverse bars.

\section{Measurements}

Holotype ( $\mathrm{mm}$ )

SVL - 57.5; TrunkL -25.9; TailL - 32.5; ArmL 13.7; LegL - 17.2; HeadL - 15.8; HeadW - 11.0; HeadH - 7.0; OrbL - 4.4; NarEye - 4.6; SnEye - 6.3; EyeEar - 6.7; INar - 1.9; IO - 5.4; Suplab - 11; Inflab - 12; CreaseL - 0.1 of height; IntNar - 0; NolO - 35; NoSC - 55; PostNas - 3; Rellab - 3 (equal); CSpurs $-5 ; 4 \mathrm{FL}-3.7 ; 4 \mathrm{FW}-0.8 ; 4 \mathrm{FscanW}-$ 1.2; 4FLam 1 - 6; 4FLam2 - 6; 4TL - 4.2; 4TW - 0.8; $4 \mathrm{Tscan} W-1.5 ; 4$ TLasm $1-7 ; 4$ TLam2 - 8; MenL/W - 1.2; TrunkL/SVL - 0.45; HeadL/SVL - 0.28; Head/ SVL - 0.19; HeadH/SVL - 0.12; ArmL/SVL - 0.24; LegL/SVL - 0.30; Tail $\%$ SVL $-56.5 \%$.

\section{Description}

A large Diplodactylus with a slight to medium build and medium-large head. Arms and legs slender and of moderate length. Tail moderately thick and short, covered by scales much larger than on dorsum. Head moderately wide and deep. Snout triangular when viewed dorsally but rounded in profile at tip. Adductor muscles of jaw large. Eyes moderately large with straight brow ridge above eve and eyes only slightly protruding above top of head. Eyelid margin begins at anterior-ventral edge of eye, then extends dorsally and anteriorily before extending posteriorly to form a protruding ridge above eye. There are $2-7$ spinose scales towards the 

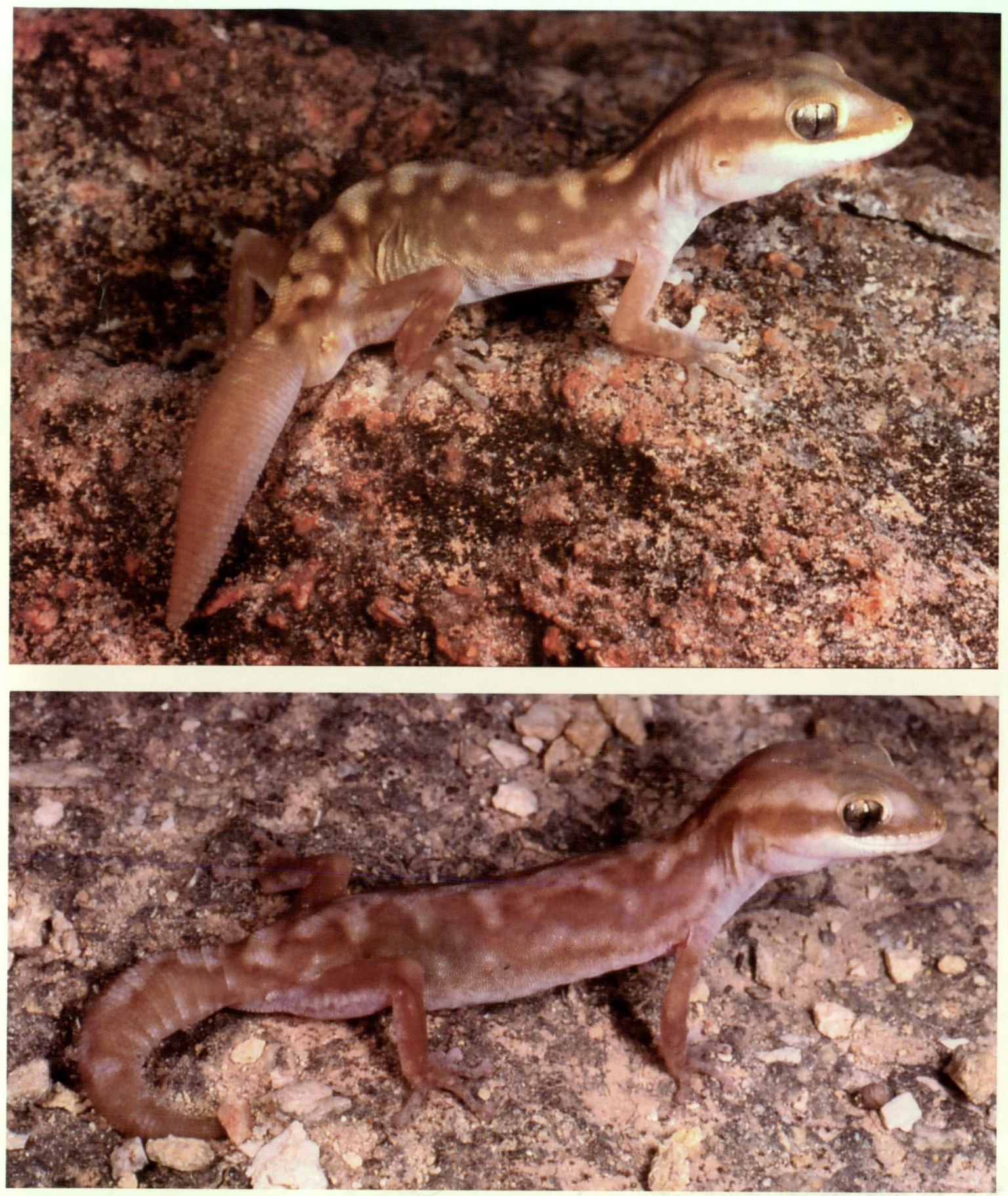

Figure 5 Male (A) and female (B) of Diplodactylus capensis sp. nov. from the Cape Range. Photographs by B. Maryan.

posterior fold of the eyelid margin. Ridge at posterior edge of eye extends anteriorly and ventrally so that the posterior ventral portion of eye socket is covered.

Usually 11 (10-12) upper and lower labial scales. Nostril surrounded by rostral, 2 supranasals, 2-4 postnasals and first labial. Second labial not lower than first. Rostral scale usually undivided $(\mathrm{N}=16)$ or at most with a small dorsal notch (3). Nostrils separated by 2 lower supranasals and usually 4 or 5 upper supranasals. Mental scale sharply triangular or lanceolate, only slightly longer than wide.
Postmentals gradually decrease in size posteriorly over 5-8 scale rows that gradually reduce to the size of the granular scales under the chin.

Scales on dorsum slightly larger than on venter. Ventral and chin scales flatter than scales on head, dorsum and tail which are more rounded. Head scales smaller than dorsal scales and chin covered with small granular scales. Limbs with small granular scales. Male cloacal spurs consist of 5-6 spinose scales arranged in 2-3 transverse rows; females have 3-10 enlarged scales (rarely spinose) where the male spurs occur. The tail is short and 


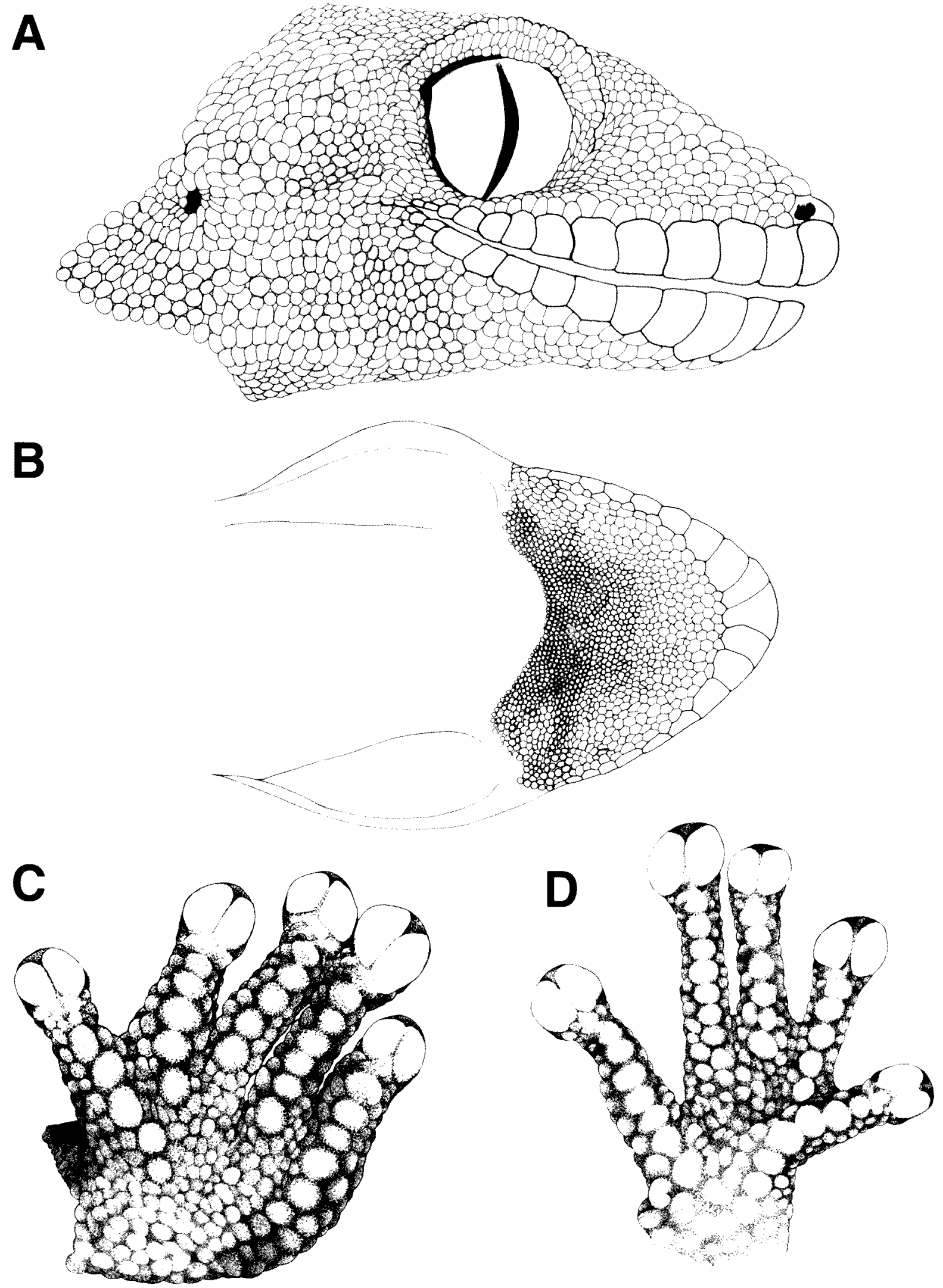

Figure 6 Holotype (WAM R154901) of Diplodactylus capensis sp. nov. Lateral (A) and ventral (B) views of head; ventral surface of hand (C) and foot (D). Drawings by C. Stevenson. 
round to slightly oblong in cross-section with a slight constriction near base. Fine granular scales cover plantar surface of manus and pes. Two enlarged terminal pads to either side of claw. The shape of the subdigital lamellae is circular, rarely tranversely oblong. Usually six unbroken subdigital lamellae on $4^{\text {th }}$ finger and eight under $4^{\text {th }}$ toe. There are 25 presacral vertebrae in the four specimens examined.

\section{Colouration}

In life the dorsal pattern consists of a light reddish-brown background which extends half-way down the flanks. The top of the head is paler than the body, with a wide pale streak extending forward from the vertebral stripe through the dorsal portion of the eye. Below this streak and above the pale upper labials is a reddish temporal streak through the ventral portion of eye. There is a creamcoloured irregular vertebral stripe with 5-8 short transverse bars to either side of midline. These travsverse bars are aligned in some animals but not in others producing an irregular serrated pattern. The stripe and transverse bars have a reddishchocolate border, but this fades on the transverse sections towards the flanks. The ventral surface and half way up the flanks is a creamy white. On original tails the dorsal vertebral stripe and transverse bar pattern continues as far as half the length of the tail before breaking up in to blotches or fading to creamy white. Regenerated tails are darker on the dorsal surface and lack the original patterns and blotches of original tails.

In very old specimens (preserved $>50 \mathrm{y}$ ), the pattern is very faint but the reddish hues and longitudinal pattern persist. In more recently preserved specimens, the rich red hues and dark borders are lost.

\section{Reproduction and ecology}

A gravid female had two eggs, one of which was $13.1 \times 7.5 \mathrm{~mm}$ (the other was damaged). Very little else is known of this species, other than its apparent preference for hard stony surfaces. Most known specimens were collected from limestone massif itself, which is extensively vegetated by hummock grass with low shrubs and trees.

\section{Etymology}

Named for the Northwest Cape of Western Australia, the region to which it is restricted.

\section{Distribution}

Diplodactylus capensis is restricted to the northern end of the Northwest Cape in Western Australia (Figure 1). Within the peninsula, it occurs on the hard rocky substrate of the limestone massif itself. There are also two records from the eastern plain near the towns of Exmouth and Learmonth.

\section{Morphological comparisons with other species}

Diplodactylus capensis differs from $D$. conspicillatus, $D$. ornatus and $D$. polyophthalmus by reddish background colour, and from $D$. conspicillatus, $D$. pulcher and $D$. Klugei by large labial scales, longer tail, mental not longer than infralabials and rostral in contact with nostril. From D. mitchelli by dorsals approximately the same size as ventrals, smaller adult body size, more robust appearance $(D$. mitchelli has a dorsoventrally compressed head and long limbs that are unique in Diplodactylus), fewer vertebrae, fewer cloacal spurs, lighter colour and uncreased rostral. It is distinguished from D. g. granarienis by greater SVL, large scale size (as measured by NolO), uncreased rostral scale, shorter tail, fewer subdigital lamellae, reddish ground colour and presence of poorly defined transverse bars on dorsum. It is distinguished from $D$. g. rex by smaller SVL and head, fewer subdigital lamellae, reddish ground colour and presence of poorly defined transverse bars on dorsum.

We found a consistent count of 25 presacral vertebrae in $D$. capensis compared to 26 in $D$. mitchelli. This is interesting given that the modal gekkotan count is 26, with a range of 23 to 29 (see Bauer et al. 1996). As only two taxa were sampled, it is not possible to determine whether the difference represents a loss or a gain, although the body shape and limb proportions of $D$. mitchelli are considerably more elongate relative to other stone geckos. Further taxon sampling of vertebral counts within the Diplodactylidae is necessary to resolve the polarity issue and to explore trends within this group.

\section{Conservation}

Diplodactylus capensis has one of the smallest distributions of an Australian gecko, although some species are only known from a single remote location (e.g., D. fulleri Storr 1978) or restricted to mountains (e.g., leaf-tailed geckos along the Great Dividing Range; Wilson and Swan 2003).

The description of $D$. capensis raises the number of reptile species endemic to the Cape Range to three. The others endemics are the blindsnake Rhamphotyhlops splendidus Aplin 1998 and the pygopod Delma tealei Maryan, Aplin and Adams 2007. In addition there are a number further reptile taxa that have isolated allopatric populations on the Cape Range (see Wilson and Swan 2003). These patterns, in addition the high levels of endemism shown by other faunal groups, underline the biogeographical uniqueness and conservation significance of the Northwest Cape. Much of the Cape Range is currently protected within the Cape Range National Park. However, the entire Northwest Cape is currently subject to degradation by large numbers of feral goats, including the 
national park. Conservation of $D$. capensis and other significant fauna on the Northwest Cape (e.g., Maryan et al. 2007) would be best achieved by increasing the size of the conservation reserve to include the entire peninsula (which is also fringed by the Ningaloo reef) and eradicating feral goats.

\section{ACKNOWLEDGEMENTS}

We thank Ken Aplin for initiating the study and obtaining the radiographs of vertebral counts, and Mark Hutchinson and Brad Maryan for providing helpful advice throughout the project including comments on earlier drafts of the manuscript. We thank Claire Stevenson for the map and drawing the figures of the holotype of $D$. capensis, and Brad Maryan for the photographs. Field work in the Cape Range was funded by a grant from Harry and Margaret Butler and genetic work was supported by grants from the Australia Pacific Science Foundation and the Australian Biological Resources Survey.

\section{REFERENCES}

Bauer, A.M., Jackman, T., Sadlier, R.A. and Whitaker, A.H. (2006). A revision of the Bavayia validiclavis group (Squamata: Gekkota: Diplodactylidae), a clade of New Caledonian geckos exhibiting microendemism. Proceedings of the California Academy of Sciences 57: 503-547.

Bauer, A.M., Russell, A.P. and Powell, G.L. (1996). The evolution of locomotor morphology in Rhoptropus (Squamata: Gekkonida): functional and phylogenetic considerations. African Journal of Hexpetology 45: 830.

Bush, B., Maryan, B., Browne-Cooper, R. and Robinson, D. (2007). Reptiles and Frogs in the Bush: Southwestern Australia. University of Western. Australia Press, Crawley, WA.

Cogger, H.G. (2000). Reptiles and Amphibians of Australia, $5^{\text {th }}$ edition. Reed New Holland, Sydney.

Han, D., Zhou, K. and Bauer, A.M. (2004). Phylogenetic relationships among gekkotan lizards inferred from $C$-mos nuclear DNA sequences and a new classification of the Gekkota. Biological Journal of theLinnean Society 83: 353-368.

Horner, P. and Adams, M. (2007). A molecular systematic assessment of species boundaries in Australian Cryptoblepharus (Reptilia, Squamata, Scincidae): a case study for the combined use of allozymes and morphology to explore cryptic biodiversity. The Beagle: in press.

Jennings, W.B., Pianka, E.R. and Donnellan, S. (2003). Systematics of the lizard family Pygopodidae with implications for the diversification of Australian temperate biotas. Systematic Biology 52: 757-780.

Kluge, A.G. (1963). Three new species of gekkonid lizard, genus Diplodactylus Gray, from Australia. Records of the Western Australian Museum 14: 545-553.
Kluge, A.G. (1967). Systematics, phylogeny, and zoogeography of the lizard genus Diplodactylus Gray (Gekkonidae). Australian Journal of Zoology 15: 1007-1108.

Maryan, B., Aplin K.P. and Adams, M. (2007). Two new species of the Delma tincta group (Squmata: Pygopodidae) from northwestern Australia. Records of the Western Australian Museum 23: 273-305.

Melville, J., Schulte, J.A. and Larson, A. (2004). A molecular study of phylogenetic relationships and the evolution of antipredator strategies in Australian Diplodactylus geckos, subgenus Strophurus. Biological Journal of the Linnean Society 82: 123-138.

Nylander, J.A.A. (2004). 'MrModelltest v2.' Program distributed by author. (Evolutionary Biology Center, Uppsala University: Uppsala, Sweden.)

Oliver, P., Hugall, A., Adams, M., Cooper, S.J.B. and Hutchinson, M. (2007). Genetic elucidation of ancient and cryptic diversity in a group of Australian geckos: the Diplodactylus vittatus complex. Molecular Phylogenetics and Evolution 44: 77-88.

Oliver, P.M., Hutchinson, M.N. and Cooper, S.J.B. (2007). Phylogenetic relationships in the lizard genus Diplodactylus Gray and resurrection of Lucasium Wermuth (Gekkota, Diplodactylidae) Australian Journal of Zoology 55: 197-210.

Pepper, M., Doughty, P. and Keogh, J.S. (2006). Molecular phylogeny and phylogeography of the Australian Diplodactylus stenodactylus (Gekkota; Reptilia) species-group based on mitochondrial and nuclear genes reveals an ancient split between Pilbara and non-Pilbara $D$. stenodactylus. Molecular Phylogenetics and Evolution 41: 539-555.

Rambaut, A. (1996). 'Se-al. Sequence alignment editor, Version 1.0 á 1.'(Department of Zoology, University of Oxford: Oxford.)

Ronquist, F. and Huelsenbeck, J.P. (2003). 'MrBayes 3: BayesianPhylogenetic Inference Under Mixed Models.' Computer program and documentation available at www.morphbank.ebc.uu.se/mrbayes

Russell, A.P. and Rosenberg, H.I. (1981). Subgeneric classification in the gekkonid genus Diplodactylus. Herpetologica 37: 86-92.

Storr, G.M. (1979). The Diplodactylus vittatus complex (Lacertilia, Gekkonidae) in Western Australia. Records of the Western Australian Museum 7: 391402.

Storr, G.M. (1988). Three new Diplodactylus (Lacertilia: Gekkonidae) from the arid zone of Australia. Records of the Western Australian Museum 14: 217-223.

Storr, G.M., Johnstone, R.E. and Smith, L.A. (1990). Lizards of Western Australia. III. Geckos and Pygopods. Western Australian Museum Press, Perth.

Swofford, D.L. (2000). 'PAUP*: Phylogenetic Analysis using Parsimony * (and other methods). Version 4.' Sinauer, Sunderland, MA.

Wilson, S. and Swan, G. (2003). A Complete Guide to Australian Reptiles. Reed New Holland, Sydney.

Manuscript accepted 22 October 2007 


\section{APPENDIX 1}

Specimens of Diplodactylus examined morphologically.

Diplodactylus capensis (non type-series material)

Males

WAM R52906, WAM R61277, WAM R81327, WAM R102844, WAM R132467.

Females

WAM R52920, WAM R52928, WAM R52929, WAM R61276.

Diplodactylus mitchelli

Males

WAM R110136, WAM R110152, WAM R113642, WAM R140406, WAM R146604, WAM R152704, WAM R154975, WAM R158095, WAM R158098, WAM R158137, WAM R170674, WAM R170686, WAM R170718.

Females

SAM R60439, WAM R84458, WAM R113618, WAM R145754, WAM R146630, WAM R146631, WAM R146632, WAM R158104, WAM R170689.

Diplodactylus granariensis granariensis

Males

WAM R72278, WAM R72361, WAM R74347, WAM R76042, WAM R84452, WAM R96573, WAM R110779, WAM R127572, WAM R134044, WAM R134106, WAM R135210, WAM R137776, WAM R144170, WAM R144551, WAM R151675, WAM R153946, WAM R153947, WAM R153948, WAM R153949, WAM R153950.

Females

WAM R136581, WAM R72718, WAM R93462, WAM R74518, WAM R72602, WAM R161181, WAM R144780, WAM R134816, WAM R132023, WAM R132278, WAM R103846, WAM R103857.

Diplodactylus granariensis rex

Males

WAM R97289,WAM R113191, WAM R132504, WAM R140415, WAM R141120, WAM R141121, WAM R144718, WAM R154934, WAM R154944, WAM R154955, WAM R154957, WAM R161164, WAM R167476, WAM R167485.

Females

WAM R100350,WAM R110702, WAM R112102, WAM R112106, WAM R136593, WAM R136619, WAM R136802, WAM R141122, WAM R145285, WAM R145448, WAM R151421, WAM R154956, WAM R167490. 
APPENDIX 2

Material of Diplodactylus examined genetically.

\begin{tabular}{|c|c|c|c|c|c|c|}
\hline Taxon & $\begin{array}{l}\text { Specimen } \\
\text { Number }\end{array}$ & Locality & State & Allozymes & mtDNA & $\begin{array}{l}\text { Genbank } \\
\text { Accession }\end{array}$ \\
\hline NW Cape & WAMR132465 & Cape Range NP & WA & Y & Y & EF532862 \\
\hline NW Cape & WAMR132466 & Cape Range NP & WA & Y & $\mathrm{N}$ & NA \\
\hline NW Cape & WAMR132467 & Cape Range NP & WA & Y & Y & NA \\
\hline NW Cape & WAMR132468 & Cape Range NP & WA & Y & Y & NA \\
\hline NW Cape & WAMR 117870 & Shothole Canyon & WA & $Y$ & $\mathrm{~N}$ & NA \\
\hline D. granariensis & WAMR153949 & Bindoon Military Training Area & WA & Y & $N$ & $\mathrm{NA}$ \\
\hline D. granariensis & WAMR153946 & Bindoon Military Training Area & WA & Y & $\mathrm{Y}$ & EF532869 \\
\hline D. granariensis & WAMR153947 & Bindoon Military Training Area & WA & $Y$ & $\mathrm{~N}$ & NA \\
\hline D. granariensis & WAMR153948 & Bindoon Military Training Area & WA & $Y$ & $\mathrm{Y}$ & NA \\
\hline D. granariensis & WAMR153950 & Bindoon Military Training Area & WA & $\mathrm{Y}$ & $\mathrm{N}$ & NA \\
\hline D. granariensis & WAMR132023 & $4 \mathrm{~km} \mathrm{W.} \mathrm{of} \mathrm{Dryandra} \mathrm{Settlement}$ & WA & $\mathrm{Y}$ & Y & NA \\
\hline D. granariensis & WAMR112106 & $20 \mathrm{~km}$ S.W Paynes Find & WA & Y & $Y$ & EF532867 \\
\hline D. granariensis & WAMR144551 & Mt Jackson & WA & $\mathrm{Y}$ & $\mathrm{Y}$ & EF532870 \\
\hline D. granariensis & WAMR112102 & $86 \mathrm{~km}$ north of Meekatharra & WA & $\mathrm{Y}$ & $\mathrm{Y}$ & EF532865 \\
\hline D. granariensis & WAMR112103 & $86 \mathrm{~km}$ north of Meekatharra & WA & Y & $\mathrm{Y}$ & EF532863 \\
\hline D. granariensis & WAMR113191 & $35 \mathrm{~km} \mathrm{~S}$ of Kumarina & WA & $\mathrm{Y}$ & $\mathrm{Y}$ & EF532866 \\
\hline D. granariensis & WAMR145285 & Hill 50 Mine, Mount Magnet & WA & Y & $\mathrm{N}$ & NA \\
\hline D. granariensis & WAMR144718 & Bungalbin & WA & $\mathrm{Y}$ & $\mathrm{Y}$ & NA \\
\hline D. granariensis & WAMR140415 & $3 \mathrm{~km}$ S. of Vivien Mine & WA & Y & Y & EF532868 \\
\hline D. granariensis & WAMR132504 & Jundee & WA & Y & $\mathrm{Y}$ & NA \\
\hline D. granariensis & WAMR141120 & 15km ENE Wildara Pinnacle & WA & $\mathrm{Y}$ & $\mathrm{N}$ & NA \\
\hline D. granariensis & WAMR141121 & 15km ENE Wildara Pinnacle & WA & $\mathrm{Y}$ & $\mathrm{N}$ & NA \\
\hline D. granariensis & WAMR141122 & 15km ENE Wildara Pinnacle & WA & $\mathrm{Y}$ & $\mathrm{N}$ & NA \\
\hline D. granariensis & WAMR127572 & Goongarrie & WA & Y & $\mathrm{Y}$ & EF532871 \\
\hline D. granariensis & WAMR145448 & Lorna Glen Station & WA & $\mathrm{Y}$ & $\mathrm{N}$ & NA \\
\hline 'Southern' & WAMR140941 & $27.5 \mathrm{~km}$ SSE Peak Eleanora & WA & Y & Y & EF532882 \\
\hline 'Southern' & SAMAR24763 & Stenhouse Bay & SA & Y & Y & EF532874 \\
\hline D. mitchelli & WAMR146604 & $198 \mathrm{~km}$ S. Port Hedland & WA & Y & $\mathrm{N}$ & NA \\
\hline D. mitchelli & WAMR146630 & 198km S. Port Hedland & WA & Y & $\mathrm{N}$ & $\mathrm{NA}$ \\
\hline D. mitchelli & WAMR146631 & 198km S. Port Hedland & WA & Y & $\mathrm{N}$ & NA \\
\hline D. mitchelli & WAMR146632 & 198km S. Port Hedland & WA & Y & Y & EF532857 \\
\hline D. mitchelli & WAMR152704 & Chichester Range & WA & Y & Y & EF532858 \\
\hline D. mitchelli & WAMR113618 & Not avaliable & WA & $Y$ & $N$ & NA \\
\hline D. mitchelli & WAMR113642 & $37 \mathrm{~km}$ NNE Auski Roadhouse & WA & Y & $Y$ & EF532857 \\
\hline D. mitchelli & SAMA R60439 & Roy Hill area & WA & $\mathrm{N}$ & Y & NA \\
\hline D. ornatus & WAMR120060 & $3 \mathrm{~km}$ E. of Greenough River Mouth & WA & $Y$ & Y & EF532859 \\
\hline D. ornatus & WAMR99299 & False Entrance Tank & WA & $Y$ & Y & EF532860 \\
\hline D. ornatus & WAM100000 & False Entrance Tank & WA & Y & $\mathrm{N}$ & NA \\
\hline D. ornatus & WAMR119295 & Wicherina Dam & WA & Y & $\mathrm{N}$ & NA \\
\hline D. pulcher & SAMAR32183 & $47 \mathrm{~km}$ N Muckera Roadhouse & SA & $X$ & $Y$ & EF532840 \\
\hline D. pulcher & WAMR120668 & Carnarvon Basin Survey & WA & $\mathrm{N}$ & Y & EF681789 \\
\hline D. pulcher & SAMA R26383 & Near Cook & SA & Y & $\mathrm{N}$ & EF532839 \\
\hline
\end{tabular}

